\title{
Pushing the Surgical Envelope
}

\author{
Rifat Latifi ${ }^{1}$
}

Accepted: 2 May 2021 / Published online: 20 May 2021

(C) Société Internationale de Chirurgie 2021

Recent decades have brought about immense and practical changes in surgery, in terms of surgical techniques and technologies; these include nanotechnologies and genomics, as well as advances in individualized surgical care. As a result, safer, more effective, albeit more complex surgical procedures can be accomplished with less morbidity and lower mortality rates. In this World Journal of Surgery Surgical Advances Symposium, we aimed to present five topics where ingenuity and innovation have changed the way surgery is practiced today.

\section{Robotic-Assisted Surgery}

Robotic-assisted surgery has become a common practice in many hospitals in the USA, and around the world. While the docking of these massive robots was once a lengthy process, and the robots themselves were viewed as unreliable, they have now evolved and altered the surgical world significantly. Due to these progressive changes, just about every surgical procedure is now being performed robotically assisted from neurosurgical procedures, orthopedic, intrathoracic, intra-abdominal, to pelvic and retroperitoneal procedures. Many laparoscopic procedures, such as inguinal and ventral hernia, and even cholecystectomy have been or are currently being replaced with a roboticassisted approach. Robotic-assisted surgery will expand in accuracy and effectiveness, and physically it will become more miniature. As the surgical world is ever evolving, we predict that most, if not all, operating rooms in the USA will contain a robot in

Rifat Latifi

Rifat_Latifi@nymc.edu; Rifat.Latifi@wmchealth.org

1 Department of Surgery, Westchester Medical Center and New York Medical College, Valhalla, NY, USA the near future. Dr. Sing et al. brings the readers of WJS a review of the current state of robotic surgery [1].

\section{Endovascular Surgery}

Endovascular surgery represents the most significant advances in vascular surgery. Drs. Blecha and Gahtan [2] bring us a state-of-the-art review of endovascular surgery in a succinct fashion. A minimally invasive approach to arterial and often venous disease with stenting has dramatically changed vascular surgery, more so than any other change in surgery with minimal morbidity. This will only continue to expand and improve, and perhaps will be used not only by vascular surgeons, intervention radiologists, neurosurgeons, neurologists, cardiologists, but also by others such as trauma general surgeons.

3. Transplant Surgery

Transplant surgery has undergone drastic and revolutionary changes. Although, there are not enough organs available for all those in need, the progress that has been made within the capabilities of organ transplant has been life altering. When a new disease called HIV emerged in the 1980s, no one would have thought that one day it would be possible for these patients to become organ donors. However, that has changed. As demonstrated in a paper by Boyarsky et al. [3], today, organs from patients with HIV and Hepatitis $\mathrm{C}$ are able transplanted into nonpositive $\mathrm{HIV}$ and $\mathrm{HCV}$ patients while minimizing the risk of spreading the disease. Overall, scientific advances in pharmacology and medicine have made it possible. These, along with other expansions of the organ pool, have undoubtedly increased the number of organs transplanted.

4. Gender Reaffirmation Surgery

Gender reaffirmation surgery has also succeeded in changing the world and image of many patients, and it 
has become routine in a number of centers of excellence around the world. This multistage, multidisciplinary surgical procedure is complex on many levels; nevertheless, it will continue to advance further. As illustrated by Jahromi et al. [4], this field will continue to progress, both from a technical as well as a medical standpoint in all aspects.

\section{Biologic Prosthesis}

Finally, one of the most common surgical procedures performed are those for hernia repair. The practice of hernia surgery has been elevated to a whole new level, particularly with advances in biologic meshes. Samson et al. [5] brought a very comprehensive review of world literature regarding this topic. This has paved the way for complex abdominal wall reconstruction (CAWR) for massive hernia, and abdominal wall domain loss even in the phase of the presence of infections with use of biologic meshes.

The world of surgery is ever is changing and advancing. As surgeons, we strive for progress so that we may become even better equipped to care for our patients, but we have realized clearly that although we still consider ourselves the captain of the surgical ship, we must work together with industry, and other innovators, for the betterment of the future of surgery. We hope that you, the reader, will enjoy them as much as we did.

\section{References}

1. Singh TP, Zaman J, Cutler J (2021) Robotic surgery: at the crossroads of a data explosion. World J Surg (in press)

2. Blecha M, Gahtan V (2020) Modern endovascular therapy. World J Surg. https://doi.org/10.1007/s00268-020-05875-7

3. Boyarsky BJ, Strauss AT, Segev DL (2021) Transplanting organs from donors with HIV or hepatitis C: the viral frontier. World $\mathrm{J}$ Surg. https://doi.org/10.1007/s00268-020-05924-1

4. Jahromi AH, Boyd LC, Schechter L (2021) an updated overview of gender dysphoria and gender affirmation surgery: what every plastic surgeon should know. World J Surg. https://doi.org/10. 1007/s00268-021-06084-6

5. Samson DJ, Gachabayov M, Latifi R (2021) Biologic mesh in surgery: a comprehensive review and meta-analysis of selected outcomes in 51 studies and 6079 patients. World J Surg. https:// doi.org/10.1007/s00268-020-05887-3

Publisher's Note Springer Nature remains neutral with regard to jurisdictional claims in published maps and institutional affiliations. 